\title{
Stationary scattering from a nonlinear network
}

\author{
Sven Gnutzmann, ${ }^{1}$ Uzy Smilansky, ${ }^{2,3}$ and Stanislav Derevyanko ${ }^{4}$ \\ ${ }^{1}$ School of Mathematical Sciences, University of Nottingham, Nottingham NG7 2RD, United Kingdom \\ ${ }^{2}$ School of Mathematics, University of Cardiff, Cardiff CF24, United Kingdom \\ ${ }^{3}$ Department Physics of Complex Systems, Weizmann Institute of Science, Rehovot, Israel \\ ${ }^{4}$ Nonlinearity and Complexity Research Group, Aston University, Birmingham, United Kingdom \\ (Received 10 November 2010; revised manuscript received 4 January 2011; published 28 March 2011)
}

\begin{abstract}
Transmission through a complex network of nonlinear one-dimensional leads is discussed by extending the stationary scattering theory on quantum graphs to the nonlinear regime. We show that the existence of cycles inside the graph leads to a large number of sharp resonances that dominate scattering. The latter resonances are then shown to be extremely sensitive to the nonlinearity and display multistability and hysteresis. This work provides a framework for the study of light propagation in complex optical networks.
\end{abstract}

DOI: 10.1103/PhysRevA.83.033831 PACS number(s): 42.81.Uv, 03.75.Kk, 05.45.Mt, 42.65.Pc

The study of quantum graphs has gained its popularity in recent years [1] not only because graphs emulate successfully complex mesoscopic and optical networks, but also because they manage to reproduce universal properties (such as level statistics, transmission fluctuations, and others) observed in generic quantum chaotic systems. Here we generalize quantum graph theory to the nonlinear domain. The theory will be applied in particular to show the effect of nonlinearity on transmission through networks of nonlinear fibers. Our model may also be used as a simple yet nontrivial model where the universal properties derived from detailed numerical computations of Bose-Einstein condensates in nonregular traps [2-7] could be further investigated.

Scattering is studied as a stationary process. The main finding is that the sharp resonances which dominate scattering in networks with complex connectivity lead to a dramatic amplification of the nonlinear effects: while nonresonant scattering hardly deviates from the predictions of the linear theory, tuning the parameters to a nearby resonance (without changing the incoming field intensity) brings the system into the nonlinear regime which is signaled by multistability and hysteresis. For this reason we revisit the theory of scattering in the linear regime and demonstrate that sharp resonances with large amplification of the incoming wave inside the system are very frequent for graphs compared to other complex (chaotic) scattering systems. The origin of this effect can be related to the topology of the graph (existence of cycles) and leads to a power-law distribution for the amplification.

\section{THE NONLINEAR SCHRÖDINGER EQUATION ON GRAPHS}

Consider a general metric graph which consists of $V$ vertices connected by $B$ internal bonds and $N$ leads to infinity, as illustrated in Fig. 1. The bonds and leads will be collectively referred to as edges. The bonds are of finite lengths $L_{b}$, $b=1, \ldots, B$ and are endowed with coordinates $x_{b} \in\left[0, L_{b}\right]$ (with a definite choice for the direction in which $x_{b}$ increases). The semi-infinite leads have the coordinate $x_{l} \in[0, \infty)$ and $x_{l}=0$ is at the vertex where the lead is attached. The wave function on the graph is a bounded piecewise continuous and differentiable function on the edges and is written collectively as

$$
\Psi(x)=\left\{\psi_{e}\left(x_{e}\right)\right\}_{e=1}^{B+N},
$$

where $\psi_{e}\left(x_{e}\right)$ is the wave functions on edge $e$. While the model can describe far more general settings, we restrict ourselves in this exploratory work to the discussion of stationary scattering. The wave function on edge $e$ satisfies the stationary nonlinear Schrödinger equation (NLSE)

$$
-\frac{d^{2} \psi_{e}}{d x_{e}^{2}}+g_{e}\left|\psi_{e}\right|^{2} \psi_{e}=E \psi_{e} .
$$

Here, $g_{e}$ is real nonlinear coupling parameter which we assume constant on each edge (but it may take different values on different edges). $E$ is taken positive throughout this work, $E=$ $k^{2}$ and $k$ reduces to the wave number (propagation constant in fiber optics) in the linear case. Setting $g_{e}=0$ on all edges will reduce our model to a standard (linear) quantum graph. Note that, for applications in fiber optics, $g|\psi|^{2} \ll E$ and the nonlinear term is a small perturbation of an otherwise linear wave equation. Then the stationary equation above describes the spatial evolution of the amplitude of a continuous wave beam rather than a wave envelope (for which one would have a nonstationary nonlinear Schrödinger equation [8]).

\section{A. The wave function on a single bond}

The solutions of (2) on a single bond can be obtained [9] by writing $\psi(x)=r(x) e^{i \theta(x)}$ (omitting the index $e$ for the moment). Then, (2) is equivalent to two coupled ordinary differential equations

$$
\begin{gathered}
\frac{d}{d x} \mathcal{H}=0 \quad \text { and } \quad \frac{d}{d x} \mathcal{L}=0, \\
\mathcal{H}=\frac{1}{2}\left(\frac{d r}{d x}\right)^{2}+\frac{r^{2}}{2}\left(\frac{d \theta}{d x}\right)^{2}+\frac{E}{2} r^{2}-\frac{g}{4} r^{4}, \\
\mathcal{L}=r^{2} \frac{d \theta}{d x} .
\end{gathered}
$$

These equations formally describe a classical particle in a central potential $V(r)=(E / 2) r^{2}-(g / 4) r^{4}$ on the twodimensional (2D) plane, where $x$ now takes the role of time. The Hamiltonian energy $\mathcal{H}$ and the angular momentum $\mathcal{L}$ are 


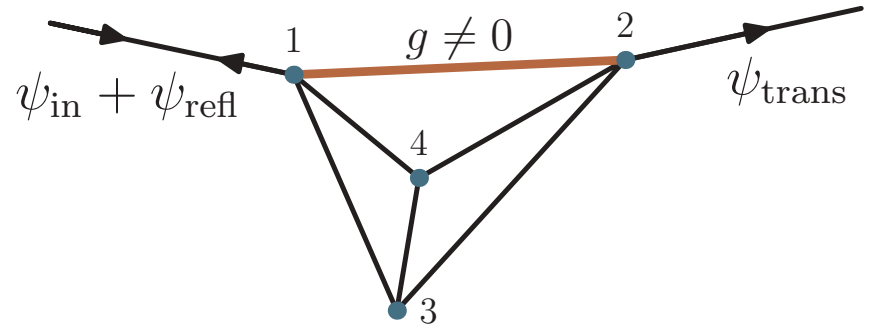

FIG. 1. (Color online) A graph with $V=4$ vertices, $B=6$ bonds and $N=2$ leads. The incoming, reflected, and transmitted waves are shown on the respective leads.

constants of motion. Note that the angular momentum (3c) reduces to the flux

$$
\mathcal{L} \equiv \operatorname{Im} \psi^{*} d \psi / d x
$$

carried by the wave function. For given values for $\mathcal{H}$ and $\mathcal{L}$ the full solution is obtained in the form of the integrals

$$
\begin{gathered}
x=\int\left(2 \mathcal{H}-2 V-\mathcal{L}^{2} / r^{2}\right)^{-1 / 2} d r, \\
\theta=\mathcal{L} \int r^{-2}\left(2 \mathcal{H}-2 V-\mathcal{L}^{2} / r^{2}\right)^{-1 / 2} d r,
\end{gathered}
$$

which can be reduced to elliptic integrals [9].

\section{B. Matching conditions at the vertices}

Two physical requirements guide our choice of matching conditions at the vertices: continuity and the conservation of flux. Consider a vertex $j$ with $v_{j}$ adjacent edges and set $x_{e}=0$ as the vertex coordinate on all edges $e$ emanating from $j$. Using the classical point-particle analog, continuity implies that at "time" $x_{e}=0$, all radii $r_{e}$ and angles $\theta_{e}$ assume the same values. Flux conservation is equivalent to angular momentum conservation:

$$
\sum_{e=1}^{v_{j}} \mathcal{L}_{e}=0
$$

There is a large family of mathematically acceptable matching conditions which satisfy the latter requirements; for example, all matching conditions that define a self-adjoint linear Schrödinger operator on the graph (see [10]) satisfy flux conservation. The matching conditions appropriate for any particular experimental setting should, in principle, be derived $a b$ initio, which is clearly a nontrivial task. Since the purpose here is to display general features of wave propagation through a nonlinear network, we chose a "minimal" set of local matching conditions commonly used in the linear case: we require continuity and that the sum over all outgoing derivatives of wave functions on edges adjacent to $j$ to be proportional to the common value $\phi_{j}$ of the wave function at the vertex:

and

$$
\psi_{e}(0)=\phi_{j}, \quad\left(e=1, \ldots, v_{j}\right)
$$

$$
\left.\sum_{e=1}^{v_{j}} \frac{d \psi_{e}}{d x_{e}}\right|_{x_{e}=0}=\lambda_{j} \phi_{j} .
$$

The constants $\lambda_{j}$ are arbitrary real parameters. In the classical particle picture, the imaginary part of this condition ensures conservation of angular momenta (6) and the real part can be expressed via

$$
\sum_{e=1}^{v_{j}} p_{e}(0)=\lambda_{j} r_{j}
$$

where $p_{e}$ is the radial momentum associated with the particle on edge $e$ and $r_{j}$ is the radial coordinate at the vertex. When $v_{j}=2$ the matching condition is equivalent to replacing the vertex by a $\delta$ potential with strength $\lambda_{j}$.

In linear quantum graphs theory, it was found useful to express the matching conditions in terms of a vertex scattering matrix $\sigma^{(j)}$, which connects the coefficients of incoming and outgoing waves [1]. Although in the nonlinear settings the lack of a superposition principle prohibits a decomposition into incoming and outgoing waves, the concept can be taken over formally. Defining

$$
\begin{aligned}
& a_{e}^{\mathrm{in}, j}=\frac{1}{2 k}\left(k \psi_{e}(0)+i \frac{d \psi(0)}{d x_{e}}\right)_{x_{e}=0}, \\
& a_{e}^{\mathrm{out}, j}=\frac{1}{2 k}\left(k \psi_{e}(0)-i \frac{d \psi(0)}{d x_{e}}\right)_{x_{e}=0},
\end{aligned}
$$

and collecting them in vectors $\mathbf{a}^{\text {in, }(j)}=\left(a_{1}^{\text {in }, j}, \ldots, a_{v}^{\text {in }, j}\right)^{T}$ and $\mathbf{a}^{\text {out },(j)}=\left(a_{1}^{\text {out }, j}, \ldots, a_{v}^{\text {out }, j}\right)^{T}$, the matching conditions become

$$
\begin{gathered}
\mathbf{a}^{\text {out },(j)}=\sigma^{(j)} \mathbf{a}^{\text {in },(j)}, \\
\sigma_{e e^{\prime}}^{(j)}=\frac{1}{v_{j}}\left(1+e^{-2 i \arctan \frac{\lambda_{j}}{v_{j} k}}\right)-\delta_{e e^{\prime}} .
\end{gathered}
$$

The flux conservation follows from the unitarity of the vertex scattering matrix and $\mathcal{L}_{e}=\operatorname{Im}\left[\psi_{e}^{*}(0) d \psi_{e}(0) / d x_{e}\right]=$ $k\left(\left|a_{e}^{\text {out }, j}\right|^{2}-\left|a_{e}^{\text {in }, j}\right|^{2}\right) \quad$ so $\quad$ that $\quad \sum_{e=1}^{v} \mathcal{L}_{e}=0 \quad$ becomes $\sum_{j}\left|\mathbf{a}^{\text {in },(j)}\right|^{2}=\sum_{j}\left|\mathbf{a}^{\text {out },(j)}\right|^{2}$-implying flux conservation. In the sequel we will assume that the vertex matching conditions (7a) and (7b) are satisfied on all vertices.

To finish the discussion of the vertex matching conditions we note that matching conditions for the time dependent NLSE on star graphs where discussed previously in $[11,12]$. The more relevant to the present work is Ref. [11] where the authors treated rigorously the case $v_{j}=2$ (see also [2]). For weak nonlinearity the vertex scattering matrix (10b) follows from their derivation.

\section{Scattering from a nonlinear network}

In the linear case the transport through a quantum graph with $N$ leads can be described by an $N \times N$ unitary scattering matrix $S(k)$ which connects incoming and outgoing amplitudes on the leads

$$
\mathbf{a}^{\text {out,leads }}=S(k) \mathbf{a}^{\text {in,leads }} .
$$

The scattering matrix $S(k)$ can be expressed explicitly in terms of the vertex scattering matrices $\sigma^{(j)}$, the bond lengths and the wave number $k$ [13] in the form

$$
S(k)=\rho+\tau_{\text {out }} \frac{1}{1-T(k) \sigma_{\text {int }}} T(k) \tau_{\text {in }} .
$$


Here $T(k)$ is a diagonal $2 B \times 2 B$ matrix with diagonal entries $e^{i k L_{b}}$ that give the phase difference of a plane wave at the two ends of the bond $b$ (each bond appears twice due to the two possible directions of a plane wave). The matrices $\rho, \sigma_{\text {int }}$, $\tau_{\text {in }}$, and $\tau_{\text {out }}$ are built up from the vertex scattering matrices $\sigma^{(j)}$. That is, $\rho$ is an $N \times N$ matrix that contains all direct scattering amplitudes (if all leads are attached to different vertices this is a diagonal matrix); the $2 B \times 2 B$ matrix $\sigma_{\text {int }}$ contains all scattering amplitudes from one (directed) bond to another inside the graph. Eventually, $\tau_{\text {in }}$ and $\tau_{\text {out }}$ are $2 B \times N$ and $N \times 2 B$ matrices that contain scattering amplitudes from the leads into the bonds and from the bonds out to the leads. They can be combined to one unitary matrix

$$
\Sigma=\left(\begin{array}{cc}
\rho & \tau_{\text {out }} \\
\tau_{\text {in }} & \sigma_{\text {int }}
\end{array}\right) .
$$

Unitarity of $\Sigma$ implies the unitarity of the scattering matrix $S(k)$. The unitarity of $S(k)$ ensures global flux conservation

$$
\left|\mathbf{a}^{\text {out,leads }}\right|^{2}=\left|\mathbf{a}^{\text {in,leads }}\right|^{2} \text {. }
$$

Moreover, in a linear system the scattering matrix $S(k)$ is independent of the incoming amplitudes $\mathbf{a}^{\text {in,leads }}$.

In the nonlinear case, transport is described by a $N$ component nonlinear scattering function

$$
\mathbf{a}^{\text {out,leads }}=\mathbf{s}\left(k, \mathbf{a}^{\text {in,leads }}\right) .
$$

Flux conservation on each vertex implies that the scattering function conserves the norm

$$
\left|\mathbf{s}\left(k, \mathbf{a}^{\text {in,leads }}\right)\right|^{2}=\left|\mathbf{a}^{\text {in,leads }}\right|^{2} .
$$

Although the general solution of the NLSE on each edge and the matching conditions are all known explicitly, it is generally not possible to solve the corresponding set of equations and obtain the scattering function $\mathbf{s}\left(k, \mathbf{a}^{\text {in,leads }}\right)$ in closed form. Therefore, we shall continue the discussion in the following section by presenting a numerical solution of a relevant example.

\section{RESONANT SCATTERING FROM A NONLINEAR NETWORK}

\section{A. A simple exemplary model}

We study the graph shown in Fig. 1. The six bond lengths were chosen by a random number generator in the interval $0<L_{b}<1$ and are rationally independent within the numerical accuracy. We have kept the same set of lengths for all numerics that are presented in this work (see [14] for the actual values). While we do not show results for different (random) choices we have checked that they lead to qualitatively equivalent results (the statistical properties that we will mention are also quantitatively equivalent).

Two linear leads (L for "left" and R for "right") are attached at vertices 1 and 2 , respectively, with $g_{\mathrm{L}}=g_{\mathrm{R}}=0$. A stationary wave with $E=k^{2}$ and intensity $I_{\text {in }}=\left|a_{L}^{\text {in }}\right|^{2}$ incident from the left lead is partially transmitted to the right lead and partially reflected:

$$
\begin{gathered}
\psi_{\mathrm{L}}\left(x_{\mathrm{L}}\right)=\psi_{\text {in }}+\psi_{\text {refl }}=a_{\mathrm{L}}^{\text {in }}\left[e^{-i k x_{\mathrm{L}}}+r\left(k, a_{\mathrm{L}}^{\text {in }}\right) e^{i k x_{\mathrm{L}}}\right], \\
\psi_{\mathrm{R}}\left(x_{\mathrm{R}}\right)=\psi_{\text {trans }}=a_{\mathrm{L}}^{\text {in }} t\left(k, a_{\mathrm{L}}^{\text {in }}\right) e^{i k x_{\mathrm{R}}} .
\end{gathered}
$$

Global gauge invariance implies that the phase of $a_{\mathrm{L}}^{\text {in }}$ can be chosen arbitrarily, so we take $a_{\mathrm{L}}^{\text {in }}=\sqrt{I_{\text {in }}} \geqslant 0$. The reflection and transmission coefficients $r\left(k, a_{\mathrm{L}}^{\text {in }}\right)$ and $t\left(k, a_{\mathrm{L}}^{\text {in }}\right)$ will be computed as functions of $k$ and $I_{\text {in }}$. In the linear case $\left(g_{e}=0\right.$ for all bonds) the reflection and transmission amplitudes are the matrix elements of a $2 \times 2$ scattering matrix $S(k)$. In the nonlinear case they form the two components of the scattering (vector valued) function $\mathbf{s}=a_{\mathrm{L}}^{\text {in }}(r(k), t(k))^{T}$. Flux conservation implies

$$
\left|r\left(k, a_{\mathrm{L}}^{\text {in }}\right)\right|^{2}+\left|t\left(k, a_{\mathrm{L}}^{\text {in }}\right)\right|^{2}=1 .
$$

In the present setting the importance of the nonlinear effects is controlled by $I_{\text {in }}$. The linear theory is obtained in the limit $I_{\text {in }} \rightarrow 0$. However, the strength of the nonlinearity will not be uniform as a function of $k$ because the intensity inside the graph structure may vary strongly, especially near resonances, as we will show below.

Using the formalism described above, the solution of the scattering problem reduces to a finite set of nonlinear equations in a high-dimensional space, which requires rather substantial computer resources. The numerical complexity can be alleviated further by considering the special case with just one nonlinear bond $\tilde{b}$, so that $g_{b}= \pm \delta_{b, \tilde{b}}$. In the present simulation we choose $\tilde{b}=(1,2)$ but we have also checked that other choices yield quantitatively similar behavior.

\section{B. Linear scattering: resonances and amplification}

The key to the understanding of the amplification of the nonlinear effects resides with the scattering in the linear regime. The upper panel of Fig. 2 shows the (linear) reflection probability $|r(k, 0)|^{2}$ as a function of the wave number $k$ for an interval of moderate wave numbers, where the typical length of a bond is about 14-16 wave lengths. Several resonances are clearly visible. Quantum graphs with incommensurate bond

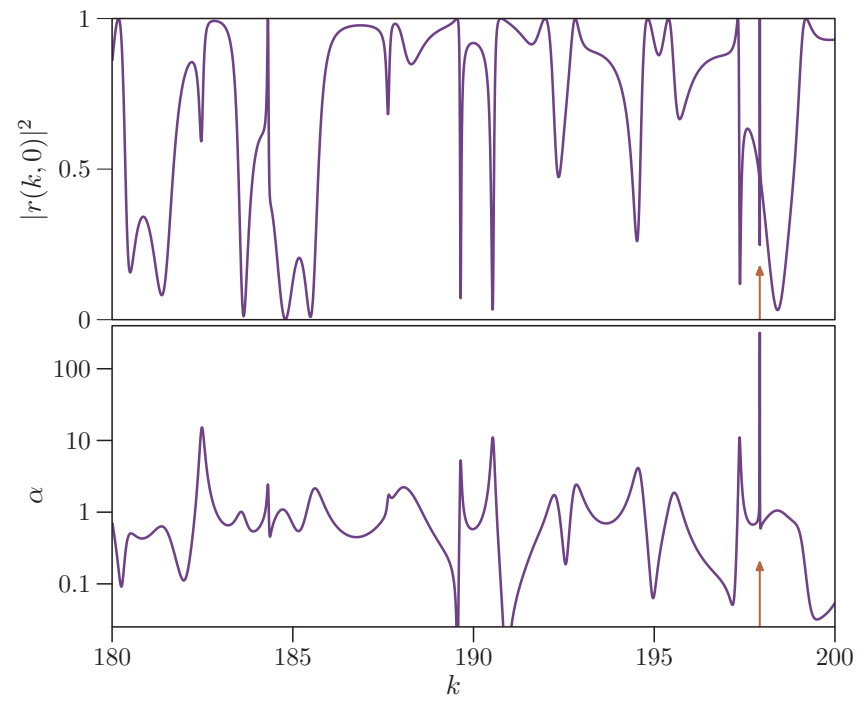

FIG. 2. (Color online) Upper panel: reflection probability $|r(k, 0)|^{2}$ for the graph depicted in Fig. 1 in the linear limit. The arrow marks the narrow resonance which is discussed in the text. Lower panel: intensity amplification factor $\alpha(k)$ in logarithmic scale in the linear limit $a_{\mathrm{L}}^{\text {in }} \rightarrow 0$ on the bond connecting vertices 1 and 2 in Fig. 1. 
lengths are a paradigm of quantum chaotic scattering [13] where the statistics of resonances (i.e., of their location and their widths) are very close to the universal predictions of random-matrix theory. This implies that the width of the resonances is distributed over a broad range of values, as illustrated by Fig. 2. The width of a single resonance is inversely proportional to the decay time of the corresponding resonant state. Narrow resonances are associated with waves which are trapped in the structure for long time, which is expressed in the stationary formalism by relatively large values of the wave function on the bonds. The lower panel in Fig. 2 shows the amplification factor

$$
\alpha(k)=\frac{\int_{0}^{L_{b}}\left|\psi_{b}\left(x_{b}\right)\right|^{2} d x_{b}}{L_{b} I_{\text {in }}}
$$

for the bond $(1,2)$ in the linear limit. While the intensity on the bond fluctuates around the incoming intensity $I_{\text {in }}$, there are also large peaks at narrow resonances. For example, near the marked sharp resonance in Fig. 2 the intensity on the bond is two orders of magnitude $(\approx 320$ times) higher than the intensity of the incoming beam. Over a larger spectral interval $(0<k<20000)$ we found several other resonances with amplification factors $\alpha>10^{5}$ and a distribution $P(\alpha)=K^{-1} \int_{0}^{K} \delta(\alpha-\alpha(k)) d k$ with a power law decay $P(\alpha) \sim \alpha^{-s}$ with $s \approx 2.85$ (see Fig. 3). The algebraic decay of this distribution is a special feature of networks, which would usually not be expected in other chaotic scattering models such as scattering through a chaotic quantum dot. To explain the difference between a network and a more general chaotic scattering system, let us go back to Eq. (12), which describes scattering through the network. Similar equations have been used to model chaotic scattering [e.g., by an average over the matrix $\Sigma$ from Eq. (13)]. In either case the factor $\left[1-T(k) \sigma_{\text {int }}\right]^{-1}$ in the second term is responsible for the resonances and one may expect large amplification factors whenever $T(k) \sigma_{\text {int }}$ has an eigenvalue near unity (an exact eigenvalue of unity indicates the existence of a bound state which is confined to the bonds). In any generic model of chaotic scattering unimodular eigenvalues of the subunitary matrix $\sigma_{\text {int }}$ (which acts on a vector of $2 B$ coefficients; one for each directed bond) are strongly suppressed. For other known examples of resonant scattering in one-dimensional nonlinear Schrödinger systems [5-7], the equivalent of $\sigma_{\text {int }}$ is one number with modulus smaller than 1 .

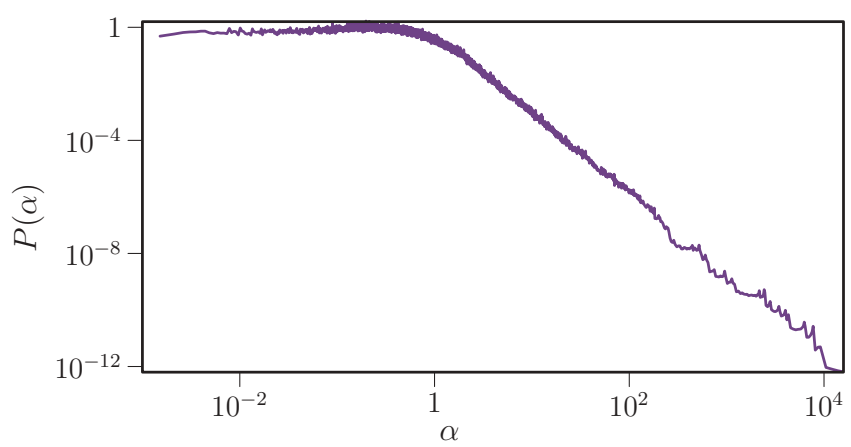

FIG. 3. (Color online) Double logarithmic plot of the numerically obtained distribution of the amplification factor on the nonlinear bond for the graph depicted in Fig. 1.
In all these models high amplification factors are either cut off or extremely rare. However, for networks with the standard vertex matching conditions (7a) and (7b) (and $\lambda_{j}=0$; see below for $\lambda_{j} \neq 0$ ) the situation is drastically different as every cycle created from the bonds of the network supports an eigenvalue unity of $\sigma_{\text {int }}$ (moreover cycles of even length support eigenvalues minus unity). In fact, let us consider a cycle that consists of three bonds $b_{1}, b_{2}$, and $b_{3}$, then corresponding eigenvector a of $\sigma_{\text {int }}$ with unit eigenvalue vanishes on all directed bonds that do not belong to the cycle and has values \pm 1 on the directed bonds that belong to the cycle (the two signs correspond to two different directions to go through the cycle). For rationally dependent bond lengths this implies that one may chose $k$ such that

$$
e^{i k L_{b_{1}}}=e^{i k L_{b_{2}}}=e^{i k L_{b_{3}}}=1,
$$

which shows the existence of embedded bound states in the continuum of scattering states. The construction is equivalent to that of perfectly scarred states (see [15]) - these scarred states vanish exactly on the vertex and, for each bond on the cycle, the bond length is an integer multiple of the wavelength $2 \pi / k$. For incommensurate bond lengths, there are no perfectly scarred states on the cycle as the condition (20) can never be met exactly. However, the mapping $k \rightarrow\left(e^{i k L_{b_{1}}}, e^{i k L_{b_{2}}}, e^{i k L_{b_{3}}}\right)$ is an ergodic flow on a three-dimensional torus; one thus finds values for the wave number $k$ which approximate condition (20) to arbitrary precision. In the exemplary model we have used for our calculation, the graph structure contains two independent cycles that both contain the nonlinear bond. The two cycles can be chosen such that they consist of three bonds.

In the above discussion we have assumed the vertex potentials $\lambda_{j}$ vanish. The vertex scattering matrices (10b) show, however, that for sufficiently high wave number $k$ these potentials are not relevant. Note also that vertex matching conditions which are entirely different from (7a) and (7b) do not necessarily have a similar distribution of narrow resonances.

\section{Implications for nonlinear scattering: multistability}

The strength of nonlinearity on the nonlinear bond may be measured by the effective parameter

$$
v=\frac{\left|g_{e}\right|}{E}|\overline{\mid}|^{2} \equiv \frac{\left|g_{e}\right|}{E L_{e}} \int_{0}^{L_{e}}\left|\psi_{e}\left(x_{e}\right)\right|^{2} d x_{e} .
$$

For a fixed incoming intensity $I_{\text {in }}$ and with $\left|g_{e}\right|=1$ the effective nonlinearity will be proportional to the amplification factor

$$
\nu(E)=\frac{1}{E} \alpha(E) I_{\text {in }} .
$$

Even if the incoming intensity is too low to induce noticeable nonlinear effects off resonance, at narrow resonances the high fields on the bonds are expected to behave in a nonlinear way. This qualitative picture is supported by the numerical simulations. For incoming intensities up to $I_{\text {in }} \approx 0.005$ the nonlinearity is either not relevant at all or can be taken into account as a perturbation for almost the entire $k$ spectrum. However, near the marked resonance the amplification by two orders of magnitude is sufficient to give rise to strong nonlinear 


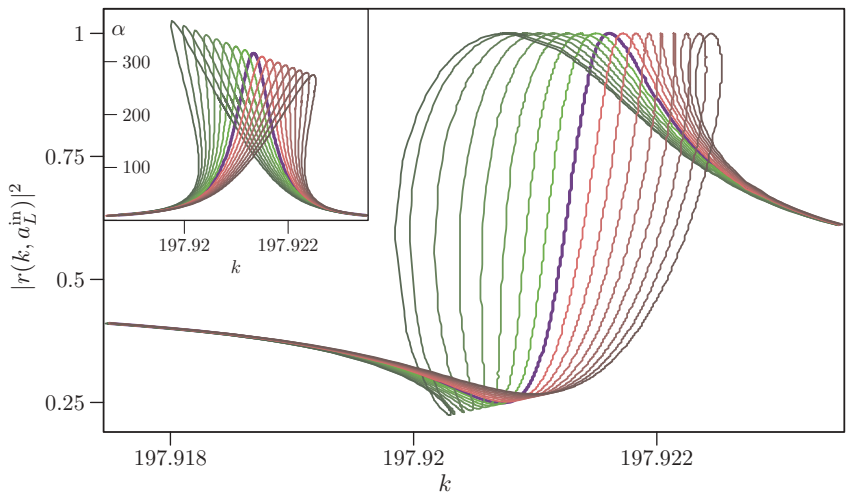

FIG. 4. (Color online) Reflection probability near the narrow resonance marked in Fig. 2. The central (blue) curve is the resonance in the linear limit. The green (red) curves left (right) from the central one correspond to 8 increasing values of the incoming intensity $\left|a_{\mathrm{L}}^{\text {in }}\right|^{2}$ (in equal steps from 0.0006 to 0.0048$)$ in the attractive $(g=1)$ and repulsive $(g=-1)$ case. The inset shows the corresponding amplification factor.

effects that cannot be described perturbatively. This is shown in figure Fig. 4, which resolves the narrow resonance in the reflection probability (and the amplification factor) for various values of the incoming intensity. In the attractive (repulsive) case the resonance moves to the left (right) as $I_{\text {in }}$ increases. However, some parts of the curve move faster than others which eventually leads to a multivalued dependence above the critical value $I_{\text {crit }}$. This implies multistability-an experiment would show hysteresis. In both the attractive and the repulsive cases the critical incoming intensity where multistability sets in is near $I_{\text {crit }} \approx 0.002$. For this incoming intensity the strength of nonlinearity inside the graph is typically (i.e., away from the resonance) on the order of $v_{\text {typ }} \sim 5 \times 10^{-8}$; at the resonance it is, however, $v_{\text {res }}=1.5 \times 10^{-5}$. These findings are similar to previous work on nonlinear resonant scattering from quantum dots [3,4] and from one-dimensional structures [5-7]. Our model generalizes the latter results by allowing for additional topological complexity.

\section{Application in nonlinear fiber optics}

The numerically found power-law distribution of the amplification factor $\alpha$ can be expected to be a generic feature (at least for similar types of vertex matching conditions). This implies that, by tuning the parameters of an experiment to a sufficiently narrow resonance, one may find arbitrarily high amplification factors and thus the nonlinearity effects of multistability and hysteresis may be observed at considerably lower incoming velocities than in our example. Nonetheless, let us translate the important parameters of our model to a fiber-network experimental setup.

For a cw optical beam propagating in a single-mode telecom fiber with the linear refraction index $n_{0}=1.5$, nonlinear
Kerr coefficient $n_{2}=2.4 \times 10^{-16} \mathrm{~cm}^{2} / \mathrm{W}$, and effective mode area $S_{\text {eff }}=50 \mu \mathrm{m}^{2}$ operating at the telecom wavelength $\lambda=$ $2 \pi c / \omega=1.55 \mu \mathrm{m}$, the strength of the nonlinearity (on the nonlinear bond) can be estimated as [8]

$$
v=\frac{8 n_{0} \omega^{2}}{c^{2} \beta^{2} S_{\text {eff }}} n_{2} P_{\mathrm{av}} .
$$

Here, $\beta$ is the propagation constant and $P_{\mathrm{av}}$ is the bondaveraged power $|\psi|^{2}$ of the beam in watts. For the purposes of the current estimate one can neglect mode dispersion of the fiber and assume $\beta \approx n_{0}(2 \pi / \lambda)$. Then, from (23) it follows that, in order to achieve the resonance level of nonlinearity $v_{\text {res }}=1.5 \times 10^{-5}$ from the numerical example considered above at the telecom wavelength $\lambda$, the required power level must be as high as $P_{\mathrm{av}} \sim 6 \mathrm{~kW}$, which is above the thresholds for stimulated Raman and Brillouin scattering for the fiber length of a few meters. This can be offset in several ways: one can consider mode dispersion and operate at lower frequencies so that the effective mode index $\beta c / \omega$ is lower, reduce the effective mode area by a factor up to 10 by changing the size of the fiber core, or else one could use highly nonlinear nonsilica fibers where the value of the nonlinear coefficient $n_{2}$ can be enhanced up to two orders of magnitude [8]. Thus, using the estimates based on the present simulation, one can expect that nonlinear effects will appear at power levels of 1/10 W.

In an experiment, varying the wave number in a controlled way may not be feasible, so let us mention that one may equivalently change the lengths of the edges in a controlled way by slowly varying the temperature. Let us also mention that multistability in scattering from nonlinear crystals has been observed experimentally [16].

\section{CONCLUSION}

To conclude, the theory presented here shows how the interplay between complex topology and nonlinearity gives rise to a pronounced amplification of nonlinear effects. We would like to stress that, while our model is highly idealized, the strong amplification of intensity near narrow resonances is a universal effect that can be expected in any linear complex network. Any coexisting nonlinearity that may be negligible off resonance will be drastically amplified at a resonance. We believe that the latter effect can be observed in actual experiments with interconnected optical fibers even though the model itself may need further adjustment to fit the details of such an experiment. Moreover we believe that NLSE on metric graphs as presented here will be a very useful paradigm system where the interplay between topology and nonlinearity can be studied qualitatively.

\section{ACKNOWLEDGMENTS}

We would like to thank Y. Silberberg, N. Davidson, P. Schlagheck, and T. Kottos for fruitful discussions.
[1] T. Kottos and U. Smilansky, Ann. Phys. 274, 76 (1999); S. Gnutzmann and U. Smilansky, Adv. Phys. 55, 527 (2006).

[2] P. Leboeuf and N. Pavloff, Phys. Rev. A 64, 033602 (2001).
[3] T. Paul, K. Richter, and P. Schlagheck, Phys. Rev. Lett. 94, 020404 (2005).

[4] T. Paul, P. Leboeuf, N. Pavloff, K. Richter, and P. Schlagheck, Phys. Rev. A 72, 063621 (2005). 
[5] K. Rapedius, D. Witthaut, and H. J. Korsch, Phys. Rev. A 73, 033608 (2006).

[6] K. Rapedius and H. J. Korsch, Phys. Rev. A 77, 063610 (2008).

[7] K. Rapedius and H. J. Korsch, J. Phys. A 41, 355001 (2008).

[8] G. P. Agrawal, Nonlinear Fiber Optics (Academic Press, New York, 2007).

[9] L. D. Carr, C. W. Clark, and W. P. Reinhardt, Phys. Rev. A 62, 063610 (2000); 62, 063611 (2000).

[10] V. Kostrykin and R. Schrader, J. Phys. A 32, 595 (1999).

[11] J. Holmer, J. Marzuola, and M. Zworski, Commun. Math. Phys. 274, 187 (2007).
[12] Z. Sobirov, D. Matrasulov, K. Sabirov, S. Sawada, and K. Nakamura, Phys. Rev. E 81, 066602 (2010).

[13] T. Kottos and U. Smilansky, Phys. Rev. Lett. 85, 968 (2000).

[14] Choice of bond lengths for the numerics presented in this work: $L_{12} \approx 0.8412, L_{13} \approx 0.4429, L_{14} \approx 0.4142, \quad L_{23} \approx 0.7483$, $L_{24} \approx 0.5137, L_{34} \approx 0.5103$ [up to ordering these are the first six numbers given by octave using the commands rand ('seed', 13); rand $(1,6)]$.

[15] H. Schanz and T. Kottos, Phys. Rev. Lett. 90, 234101 (2003).

[16] P. E. Barclay, K. Srinivasan, and O. Painter, Opt. Express 13, 801 (2005). 